\title{
Periodontal disease - risk factors and treatment options
}

2018 Theme article: Oral manifestations of systemic disease

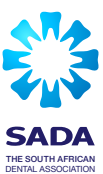

SADJ October 2018, Vol. 73 No. 9 p571 - p575

TK Madiba', A Bhayat ${ }^{2}$

\section{SUMMARY}

Periodontal disease (PD) encompasses both gingivitis and periodontitis. Both are initiated by plaque and are influenced by the immune and inflammatory responses of each individual. In addition, PD is modified by several risk factors including smoking, medications, alcohol, age, gender and systemic diseases.

Gingivitis affects $50-90 \%$ of adults worldwide and is reversible by simple, effective oral hygiene and lifestyle changes. Between 10-15\% of the global adult population suffer from progressive periodontitis, which if left unattended, results in halitosis, pain and loss of teeth.

As dental plaque is the principal etiological factor in the pathogenesis of $\mathrm{PD}$, effective oral hygiene and plaque removal is the most important strategy in the prevention of this disease. There is also evidence that PD has several modifiable risk factors in common with certain non-communicable chronic diseases like diabetes. Therefore, to prevent PD, the approach of controlling the common risk factors could be an effective strategy.

Potential risk-factor entry points are reduction of tobacco use, reduction in consumption of harmful levels of alcohol, a healthy diet and good nutrition and improvement of personal hygiene. Whilst PD is not contagious it can become extremely common and debilitating, given the ideal environment. This paper discusses the risk factors and identifies options by which PD can be prevented and reduced.

\section{INTRODUCTION}

Periodontal disease (PD) encompasses a cluster of diseases that result in inflammatory responses and chronic destruction of the tissues that surround and support the teeth, namely the gingiva, periodontal ligament, cementum and alveolar bone (collectively referred to as the "periodontium"). ${ }^{1,2}$ It therefore refers to both gingivitis and periodontitis. ${ }^{1,2}$

\section{Author affiliations:}

1. Thomas K. Madiba, B.Dent Ther, BDS, DHSM, MChD (Community Dentistry), Department of Community Dentistry, School of Dentistry, University of Pretoria, Pretoria, South Africa.

2. Ahmed Bhayat, BDS, MSc, MPH, M Dent (Community Dentistry), Department of Community Dentistry, School of Dentistry, University of Pretoria, Pretoria, South Africa.

Corresponding author: Thomas Madiba

Department of Community Dentistry, School of Dentistry, University of

Pretoria, Pretoria, South Africa.

Tel: +27 (0)12 3192417 Email: thommy.madiba@up.ac.za

\author{
ACRONYMS \\ LGE: $\quad$ Linear Gingival Erythema \\ NUG: $\quad$ Necrotising Ulcerative Gingivitis \\ NUP: $\quad$ Necrotising Ulcerative Periodontitis \\ PD: $\quad$ Periodontal Disease \\ SES: $\quad$ Socioeconomic Status
}

Gingivitis is an inflammatory condition of the soft tissues (gingiva) surrounding the teeth whilst periodontitis involves the destruction of the supporting structures of the teeth and periodontium. ${ }^{2}$

Clinical signs of a healthy periodontium include:

maintenance of a functional periodontal attachment level, minimal or no recession with no loss of interproximal bone; and, where present, functional dental implants, all in the absence of inflammation. ${ }^{3}$ (Fig. 1).

Both gingivitis and periodontitis are initiated by plaque and are influenced by the immune and inflammatory responses of the individual. Both conditions are modified by several factors including smoking, medication, age and systemic diseases.

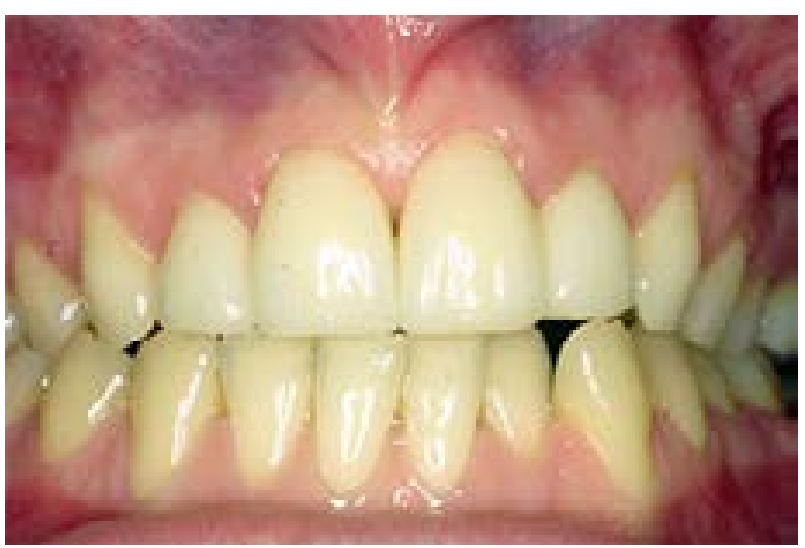

Figure 1. Healthy gums.

Gingivitis affects 50-90\% of adults worldwide (Fig. 2) and is readily reversible by simple, effective good oral hygiene and lifestyle changes. ${ }^{4}$

Gingivitis can be defined as the presence of gingival inflammation, whereby the gum can appear reddened, swollen, and may easily bleed, but without loss of connective tissue attachment. 
Periodontitis can be defined as the presence of gingival inflammation at sites where there has been a pathological loss of attachment. This loss of attachment contributes to pocket formation. The rate of progression of periodontitis is neither predictable nor steady.

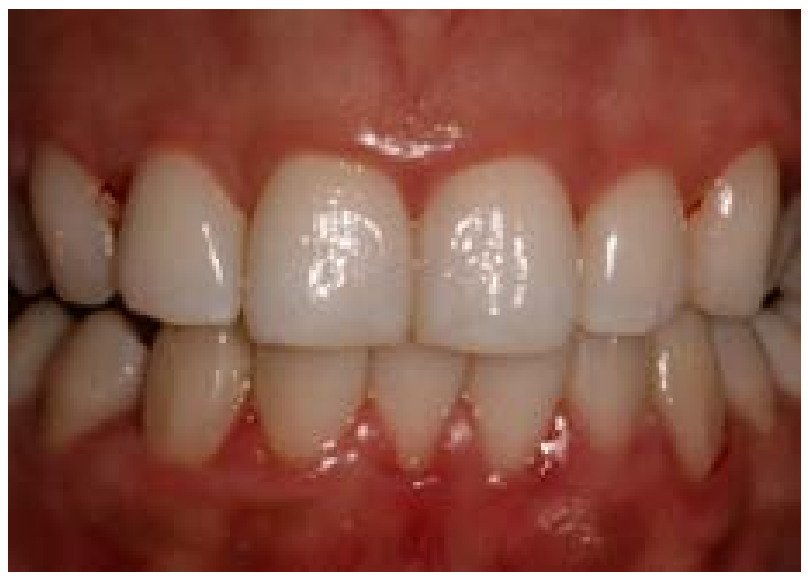

Figure 2. Mild Gingivitis.

The disease is considered to progress in relatively short episodes of rapid tissue destruction, sometimes followed by some repair, and mostly by prolonged periods of dormancy (Fig. 3). ${ }^{3}$

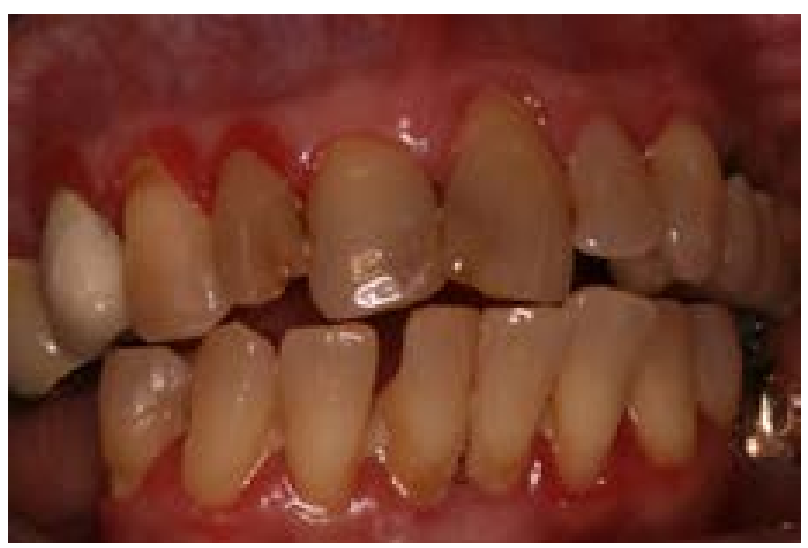

Figure 3. Periodontitis.

Severe periodontitis can result in loosening of teeth, occasional pain and discomfort, impaired mastication, and eventual tooth loss. ${ }^{4}$ Periodontitis does not affect all teeth evenly but has individual and site predilection; approximately $10-15 \%$ of the global adult population suffer from progressive periodontitis. ${ }^{2}$

\section{Role of plaque in periodontal disease}

Dental plaque is the principal etiological factor in the pathogenesis of PD. The presence of plaque is necessary, but is not of itself sufficient, for periodontal disease to occur.

A variety of disease patterns, both between different individuals and between different sites in the mouth within the same individual can be related to: the host response, the modifying effect of various risk factors and the bacterial attack from dental plaque, involving mostly Gram-negative, anaerobic bacteria growing in subgingival sites. ${ }^{1,3}$
Calcified plaque (calculus) does not have a major role in the pathogenesis of periodontal disease, although it does act as a 'retention web' for bacteria and interferes with the ability to perform personal oral hygiene.

Studies have shown that microorganisms quickly colonize clean tooth surfaces after cessation of oral hygiene procedures and that within a few days microscopic and clinical signs of gingivitis can be observed. ${ }^{5}$ At this point these changes can be reversed, provided the individual resumes adequate tooth cleaning procedures.

Gingivitis is a result of the microorganisms within the plaque releasing products that induce tissue inflammation. Most individuals develop clinical signs of gingivitis after 10-20 days of plaque accumulation. ${ }^{5}$ It has been noted that not all patients will develop periodontitis following gingivitis and therefore those who do must have a unique response to microbial plaque.

The teeth specificity and predilection in periodontal disease probably localise to the sites of retention of plaque where oral hygiene is inadequate or in areas of calculus formation, restorative overhangs or poor margins of crowns. In normal situations, more than six months may pass before the lesion of gingivitis changes to periodontits. ${ }^{6}$

\section{Microbiology of periodontal disease}

About 300 to 400 bacterial species are found in sub-gingival plaque but only about 10 to 20 species may play a role in the pathogenesis of destructive PD. ${ }^{2}$ It is only those species that are able to colonize that result in damage to periodontal tissues. Colonization requires the ability to attach to periodontal tissues, to multiply, to compete with other microbes within the oral environment and the resilience to survive the host defense mechanisms.

The microbes involved with PD are largely gram negative anaerobic bacilli, as mentioned, with some anaerobic cocci and a large quantity of anaerobic spirochetes. The main organisms linked with deep destructive periodontal lesions are Porphyromonas gingivalis, Prevotella inter-media, Bacteroides forsythus, Actinobacillus actinomycetumcomitans, and Treponema denticola. ${ }^{2}$

\section{Risk factors associated with periodontitis}

Periodontal disease is considered to have multiple risk factors. According to one author the term "risk factor refers to an aspect of personal behaviour or lifestyle, an environmental exposure, or inherited characteristics, which on the basis of epidemiological evidence is known to be associated with a health related condition".7 Risk factors therefore are part of the causal chain for a particular disease or can lead to an exposure of an individual to a disease and therefore the presence of risk factors implies a direct increase in the probability of the disease occurring.

Destructive periodontal disease is a consequence of the interaction of genetic, environmental, host and microbial factors. ${ }^{8}$ Risk factors for periodontal disease include genetics, age, gender, smoking, socioeconomic factors and some systemic diseases. 
Age

The prevalence of periodontal disease is seen to increase with age, while the extent and severity also increases with advancing age. ${ }^{9}$

However, it is not clear whether becoming older is related to an increased susceptibility to periodontal disease or whether the cumulative effects of disease over a lifetime may explain the increased prevalence of disease in older people. ${ }^{2}$

Some authors suggest that up until 70 years of age the rate of periodontal destruction is the same throughout adulthood; age per se is not a risk factor for people under $70 . .^{10,11}$

In South Africa life expectancy increased from 59 years in 2000 to 63.6 years in 2016. ${ }^{12}$ This increase in life expectancy is considered mainly due to the mass roll out of Antiretroviral (ARV) treatment for HIV and its positive consequences.

The increased life expectancy would result in an increased geriatric population requiring oral health treatment especially for periodontal diseases.

\section{Socioeconomic status}

A possible relationship between PD and socioeconomic status (SES) was found in several studies. ${ }^{13-15}$ Gingival condition is directly related to SES with evidence of poor gingival health and is more prevalent in persons from a low SES.

The relationship between SES and periodontitis is less direct. It can be certain that gingival health is better among individuals with higher education and with a more secure income. ${ }^{16}$ South Africa has a high unemployment rate of around $27 \%$ and coupled to this is a high disparity between the rich and the poor. ${ }^{17,18}$

These factors combined with poor education and low levels of knowledge all indicate that the prevalence of periodontal diseases will increase in the next few years, placing an added burden on the public oral health sector.

\section{Race and gender}

Destructive periodontitis is consistently more prevalent in males than females which could be due to lifestyle choices of males which include an increased alcohol and smoking consumption. ${ }^{9}$

PD also has been reported to be more prevalent amongst blacks than whites with a Brazilian study reporting that groups of blacks have a three times higher risk of periodontal destruction compared with whites of the same age cohort. ${ }^{14}$

This could be due to lifestyle choices and genetic factors and may be applicable to similar South African populations. The distribution of PD within countries also differs according to race or ethnic group regarding prevalence and severity. ${ }^{19}$
South Africa comprises 81\% Black African and just over $51 \%$ of all South Africans are females. ${ }^{20}$ These factors also impact on the prevalence of PDs and clinicians should be aware of these risk factors when examining and treating their patients.

\section{Smoking}

A consistent, positive association between smoking and loss of periodontal attachment has been reported and confirmed in many studies. ${ }^{21,} 22$ Smoking alone accounts for more than $50 \%$ of PD cases.

The prevalence of smoking is higher in the uneducated, poorer communities and low-income earners compared with their more affluent and educated counterparts. ${ }^{9}$ Other studies have reported that smokers were five times more likely to have a periodontal emergency compared with non-smokers. ${ }^{23}$

The disease is more prevalent, extensive and severe among current smokers, and occurs least amongst those who have never smoked. It has been proven that those who smoke over many years have considerably higher periodontal disease rates, and that this occurs even with cannabis smoking, suggesting that it is the smoking, rather than any specific characteristic of tobacco, that is responsible. ${ }^{24}$

Although South Africa has seen a decrease in the national tobacco consumption, tobacco use remains prevalent in certain underprivileged and marginalized communities. ${ }^{25}$

Added to this, studies have reported an increase of electronic cigarette and water pipe smoking and long term research is required on the effects of these innovative habits on the oral cavity. ${ }^{26}$

\section{Genetics}

There is a growing body of evidence from studies that genetic factors predispose individuals to periodontal disease. This is seen especially in the rare and more severe forms of periodontitis like early onset periodontitis, now classified as aggressive periodontitis, ${ }^{27}$ where family studies have provided good evidence for a prominent genetic role. ${ }^{28,29}$ A gene mutation for pre-pubertal periodontitis has been identified. ${ }^{30}$

\section{Systemic disease}

Systemic disease can adversely affect host defense systems and therefore can act as risk factors for PD. ${ }^{2}$ Among the associations observed between oral health status and chronic systemic diseases, the link between PD and diabetes mellitus is the most consistent. ${ }^{31}$

Periodontal diseases are well established as a complication of diabetes, and have been considered the sixth most common complication of diabetes. ${ }^{32,33}$

In a study of Brazilian individuals with poorly controlled type 2 diabetes, significantly higher levels of periodontal pockets and loss of attachment were found compared with controls. ${ }^{34}$ 
Although HIV disease has a relatively minor effect on the progression of chronic periodontitis compared with other pathogenic factors, patients who are HIV-positive and immunosuppressed can present with distinctive forms of necrotising gingivitis and periodontitis. ${ }^{35}$

Diseases of the oral cavity strongly associated with HIV are: linear gingival erythema (LGE), necrotising ulcerative gingivitis (NUG) and necrotising ulcerative periodontitis (NUP). It has been proven that the presence of NUP and NUG may offer a significant diagnostic, as well as a prognostic, value. ${ }^{36}$

\section{Periodontitis and stress}

It is well known that cardiovascular disease, diabetes, and other chronic diseases are related to psychosocial factors, but there is also evidence that stress is linked to periodontal disease.

Stressful life events, and marital problems are associated with PD, possibly through physiologic responses that increase susceptibility and reduce the immune response..$^{9,19}$

\section{Periodontitis and pregnancy}

Studies have demonstrated that PD have been shown to increase the risk of adverse pregnancy outcomes such as premature birth and low birth weight. ${ }^{37-39}$ Uterine contractions are stimulated by oxytocin, which is produced by the hypothalamus and by prostaglandins produced by the placenta. This process normally occurs in the third trimester and leads to birth. However, chronic infection can stimulate the inflammatory process, which leads to elevated amniotic levels of prostaglandins, TNF- $\alpha$, Interleukin-1 and -6 . These mediators then lead to premature rupture of membranes and pre-term labour. Other work has suggested that periodontal pathogens may travel from the gingival sulcus to the placenta and stimulate preterm birth. ${ }^{40}$

\section{Alcohol}

High alcohol consumption increases the risk of a wide variety of conditions such as increased blood pressure, liver cirrhosis, cardiovascular disease, diabetes, and cancers of the mouth. ${ }^{19}$ Recent research also indicates that excessive alcohol consumption is associated with increased severity of periodontal disease. ${ }^{41,42}$

Alcohol consumption, tobacco use, and unhealthy diet commonly go together. People who consume tobacco are more likely to drink alcohol and eat a diet high in fats and sugars but low in fiber and polyunsaturated fatty acids. Those who have a high consumption of tobacco and alcohol are thus more likely to be at a higher risk of severe periodontal disease and oral cancer. ${ }^{19}$

\section{Local risk factors}

Any plaque retentive feature such as restoration overhangs or deficiencies, may contribute to the local risk of periodontal disease. ${ }^{2}$

\section{CONCLUSION}

Risk factors work to change the susceptibility or resistance of individuals to the disease. Risk factors for periodontal disease can be both systemic and local, such as smoking; medical conditions, poorly controlled diabetes, possibly obesity and stress play a significant role in the initiation and progression of PD.

The modification of these risk factors plays a strategic role in the management of periodontal disease, accepting of course that some, such as race or genetics, cannot be changed. The identification of high-risk patients is therefore essential in the ultimate management and treatment of PDs.

As has been demonstrated, periodontal disease is highly linked to systemic diseases such as diabetes and HIV. There is also evidence that periodontal disease has several modifiable risk factors in common with certain non-communicable chronic diseases and therefore to prevent periodontal disease one can use the common risk factor approach.

It is therefore essential that clinicians adopt a holistic and systemic approach to identify high risk patients and to recommend behaviour and lifestyle changes to attain the common goal of preventing and managing PDs.

\section{References}

1. Xiong X, Buekens $P$, Fraser W, Beck J, Offenbacher S. Periodontal disease and adverse pregnancy outcomes: a systematic review. BJOG. 2006;113(2):135-43.

2. Kinane DF. Causation and pathogenesis of periodontal disease. Periodontol 2000. 2001;25(1):8-20.

3. Beirne PV, Worthington HV, Clarkson JE. Routine scale and polish for periodontal health in adults. The Cochrane Library. 2007.

4. Pihlstrom BL, Michalowicz BS, Johnson NW. Periodontal diseases. The Lancet. 2005;366(9499):1809-20.

5. Weijden G, Timmerman M, Danser M, Nijboer A, Saxton $\mathrm{C}$, Velden $\mathrm{U}$, et al. Effect of pre-experimental maintenance care duration on the development of gingivitis in a partial mouth experimental gingivitis model. J Periodontal Res. 1994;29(3):168-73.

6. Brecx M, Fröhlicher I, Gehr P, Lang N. Stereological observations on long-term experimental gingivitis in man. J Clin Periodontol. 1988:15(10):621-7.

7. Last JM. A dictionary of epidemiology. Oxford University Press, 1995. McMichael AJ, Anderson HR, Brunekreef B, Cohen AJ. Inappropriate use of daily mortality analyses to estimate longerterm mortality effects of air pollution Int $\mathrm{J}$ Epidemiol. 1998:27:450-3.

8. Zambon JJ. Periodontal diseases: microbial factors. Ann Periodontol. 1996;1(1):879-925.

9. Watt RG, Petersen PE. Periodontal health through public health-the case for oral health promotion. Periodontol 2000. 2012;60(1):147-55.

10. Holm-Pedersen P, Agerbæk N, Theilade E. Experimental gingivitis in young and elderly individuals. J Clin Periodontol. 1975;2(1):14-24.

11. Machtie E, Dunford R, Grossi S, Genco R. Cumulative nature of periodontal attachment loss. J Periodontal Res. 1994;29(5):361-4.

12. World Health Organization. World Health Statistics : monitoring health for the Sustainable Developmental Goals(SDGs). 2018. Licence: CC BY-NC-SA 3.0 IGO.Geneva 
13. Beck JD, Koch GG, Rozier RG, Tudor GE. Prevalence and risk indicators for periodontal attachment loss in a population of older community-dwelling blacks and whites. J Periodontol. 1990;61(8):521-8.

14. Gilbert GH. Racial and socioeconomic disparities in health from population-based research to practice-based research: the example of oral health. J Dent Educ. 2005;69(9):1003-14.

15. Susin C, Oppermann RV, Haugejorden O, Albandar JM. Tooth loss and associated risk indicators in an adult urban population from south Brazil. Acta Odontol Scand. 2005;63(2):85-93.

16. AlJehani YA. Risk factors of periodontal disease: review of the literature. Int J Dent. 2014;2014.

17. Moalusi T. Statistics South Africa. Youth unemployment in SA increasing - where to from here? HR Future. 2018;2018(Jul 2018):8-9. accessed 17 October 2018. Available at http:// www.statssa.gov.za/?p=11129

18. Ataguba JE, Akazili J, Mclntyre D. Socioeconomic-related health inequality in South Africa: evidence from Genera Household Surveys. Int J Equity Health. 2011;10(1):48.

19. Petersen PE, Ogawa H. Strengthening the prevention of periodontal disease: the WHO approach. J Periodontol. 2005;76(12):2187-93.

20. Statistics South Africa. Mid-year population estimates. 2017. assesed 17 October 2018. available at. http://www.statssa. gov.za/publications/P0302/P03022018.pdf

21. Haber J. Smoking is a major risk factor for periodontitis. Curr Opin Periodontol. 1993:12-8.

22. Haber J, Wattles J, Crowley M, Mandell R, Joshipura K, Kent $\mathrm{RL}$. Evidence for cigarette smoking as a major risk factor for periodontitis. J Periodontol. 1993;64(1):16-23.

23. Deutsch WM. Dental events during periods of isolation in the US submarine force. Mil Med. 2008;173(Supplement_1):29-37.

24. Thomson WM, Sheiham A, Spencer AJ. Sociobehaviora aspects of periodontal disease. Periodontol 2000. 2012;60(1):54-63.

25. Teare J, Naicker N, Albers P, Mathee A. Prevalence of tobacco use in selected Johannesburg suburbs. S Afr Med J. 2018;108(1):40-4.

26. Ramôa C, Eissenberg T, Sahingur S. Increasing popularity of waterpipe tobacco smoking and electronic cigarette use: Implications for oral healthcare. J Periodontal Res. 2017; 52(5):813-23.

27. Wiebe CB, Putnins EE. The periodontal disease classification system of the American Academy of Periodontology - an update. J Can Dent Assoc. 2000;66(11):594-9.

28. Hassell TM, Harris EL. Genetic influences in caries and periodontal diseases. Crit Rev Oral Biol Medicine. 1995;6(4):319-42.
29. Michalowicz BS. Genetic and heritable risk factors in periodontal disease. J Periodontol. 1994;65(5s):479-88.

30. Hart T, Hart P, Michalec M, Zhang Y, Marazita M, Cooper M, et al. Localisation of a gene for prepubertal periodontitis to chromosome $11 \mathrm{q} 14$ and identification of a cathepsin $\mathrm{C}$ gene mutation. J Med Genet. 2000;37(2):95-101.

31. GrossiSG, Genco RJ. Periodontal disease and diabetes mellitus: a two-way relationship. Ann Periodontol. 1998;3(1):51-61.

32. Grossi SG, Skrepcinski FB, DeCaro T, Zambon JJ, Cummins $D$, Genco RJ. Response to periodontal therapy in diabetics and smokers. J Periodontol. 1996;67(10s):1094-102.

33. Taylor GW, Burt BA, Becker MP, Genco RJ, Shlossman M. Glycemic control and alveolar bone loss progression in type 2 diabetes. Ann Periodontol. 1998;3(1):30-9.

34. Novaes Jr AB, Gutierrez FG, Novaes AB. Periodontal disease progression in type II non-insulin-dependent diabetes mellitus patients (NIDDM). Part I-Probing pocket depth and clinical attachment. Braz Dent J. 1996;7(2):65-73.

35. Robinson PG, Adegboye A, Rowland R, Yeung S, Johnson N. Periodontal diseases and HIV infection. Oral Dis. 2002;8(s2):144-50.

36. Mataftsi M, Skoura L, Sakellari D. HIV infection and periodontal diseases: an overview of the post-HAART era. Oral Dis. 2011;17(1):13-25.

37. Offenbacher S, Katz V, Fertik G, Collins J, Boyd D, Maynor G, et al. Periodontal infection as a possible risk factor for preterm low birth weight. J Periodontol. 1996;67(10s):1103-13.

38. Jeffcoat MK, Geurs NC, Reddy MS, Cliver SP, Goldenberg RL, Hauth JC. Periodontal infection and preterm birth: results of a prospective study. J Am Dent Assoc. 2001;132(7):875-80.

39. Scannapieco FA, Bush RB, Paju S. Periodontal disease as a risk factor for adverse pregnancy outcomes. A systematic review. Ann Periodontol. 2003;8(1):70-8.

40. Gurenlian J. Inflammation: the relationship between oral health and systemic disease. Dental Assistant (Chicago, III: 1994). 2009;78(2):8.

41. Tezal M, Grossi SG, Ho AW, Genco RJ. The effect of alcohol consumption on periodontal disease. J Periodontol. 2001;72(2):183-9.

42. Pitiphat W, Merchant A, Rimm E, Joshipura K. Alcohol consumption increases periodontitis risk. J Dent Res. 2003; 82(7):509-13. 